\title{
The utility of Coccidioides antigen and antibody detection in cerebrospinal fluid in the diagnosis of canine central nervous system coccidioidomycosis
} \author{
Lisa F. Shubitz, DVM ${ }^{1 *}$ \\ IThe Valley Fever Center for Excellence, University of Arizona, Tucson, AZ \\ ${ }^{2}$ Veterinary Specialty Center of Tucson, Tucson, AZ \\ ${ }^{3}$ MiraVista Diagnostics, Indianapolis, IN \\ *Corresponding author: Dr. Shubitz (Ifshubit@arizona.edu).
}

Christine D. Butkiewicz, DVM, MPH ${ }^{1}$; Cody J. Alcott, DVM ${ }^{2}$; Janelle Renschler, DVM, PhD ${ }^{3}$; Lawrence J. Wheat, MD ${ }^{3}$;

\section{OBJECTIVE}

To evaluate the utility of enzyme immunoassays (EIAs) for the detection of Coccidioides antigen and antibody in CSF in the diagnosis of CNS coccidioidomycosis in dogs.

\section{ANIMALS}

5 I dogs evaluated for CNS disease in a single specialty center in Tucson in 2016.

\section{PROCEDURES}

Excess CSF after routine analysis was banked after collection from dogs presented to the neurology service. Samples were tested by EIA for presence of Coccidioides antigen and antibody. Clinical data were collected from medical records retrospectively.

\section{RESULTS}

22 dogs were diagnosed with CNS coccidioidomycosis (CCM) or another neurologic disease (non-CCM). These groups of dogs overlapped in the presenting complaints, MRI results, and routine CSF analysis results. Four dogs, all with CCM, had positive antigen EIA results. With clinical diagnosis used as the reference standard, CSF antigen testing had low sensitivity (20\%) but high specificity (I00\%) for diagnosis of CCM. Ten dogs with CCM and 4 dogs with other diagnoses had antibody detected in CSF by EIA. Sensitivity of CSF antibody testing was $46 \%$, specificity was $86 \%$, and positive and negative predictive values for the study population were $71 \%$ and $68 \%$, respectively.

\section{CLINICAL RELEVANCE}

Diagnosis of CNS coccidioidomycosis in dogs in an endemic region was hampered by overlap of clinical signs with other neurologic disorders and the low sensitivity of confirmatory diagnostics. The evaluated Coccidioides-specific ElAs performed on CSF can aid in the diagnosis. A prospective study is warranted to corroborate and refine these preliminary findings

Ooccidioidomycosis, a systemic fungal infection
immitis and Coccidioides posadasii, is a common
condition in dogs in Arizona, southern and central
California, and other parts of the southwestern US.
Although it most commonly presents as a fungal pneu-
monia, the infection can disseminate to other sites,
including the CNS. ${ }^{2-4}$ Historically, the most common
clinical sign related to coccidioidal brain infection in
dogs is seizures, ${ }^{2,5,6}$ and the most common imaging,
gross, and histopathological findings are 1 or more
granulomatous mass lesions with coccidioidal spher-
ules. ${ }^{3,7}$ However, Spoor et al ${ }^{8}$ reported coccidioidal
encephalopathy diagnosed by MRI, positive results of
anti-Coccidioides serologic tests, and response to an-
tifungal treatment. ${ }^{8}$ Davidson et al ${ }^{3}$ also reported that
MRI demonstrated meningoencephalitis in dogs with
CNS coccidioidomycosis, with dogs having a wider range of clinical signs than seizures alone. There is overlap of the known clinical and diagnostic findings in dogs with CNS coccidioidomycosis and other neurologic diseases, including neoplasia, toxins, and inflammatory diseases.

Diagnosis of dogs with CNS coccidioidomycosis is hindered by infrequent definitive identification of the pathogen in affected anatomic sites while the patient is alive due to cost, invasiveness of sample collection, and low rate of identifying organisms in CSF or other CNS tissue. Diagnostic criteria are well established for coccidioidal meningitis in humans. These criteria include symptoms of meningitis, coupled with serum antibody detection or definitive diagnosis of coccidioidomycosis in another site, and CSF abnormalities defined as elevated protein, decreased glucose, and pleocytosis., 4 Cerebrospinal fluid abnormalities and presence of antiCoccidioides antibody have not been comprehensively 
reported for dogs with CNS coccidioidomycosis, and diagnosis is typically based on clinical signs and positive serology results or a history of coccidioidomycosis in dogs living in regions endemic for the disease. ${ }^{7,10}$

Fungal culture is a low-yield (7\%) diagnostic test for Coccidioides infection in humans, ${ }^{11}$ and identification of any etiologic agent in the CSF in dogs is uncommon as well (2\%). ${ }^{12}$ Other Coccidioides-specific CSF testing has been studied in humans. ${ }^{13-15}$ In 1 such study, ${ }^{11}$ anti-Coccidioides antibody was detected by enzyme immunoassay (EIA) in the CSF of $85 \%$ of patients diagnosed with Coccidioides meningitis. Coccidioides antigen detection by EIA was found to have a sensitivity of $93 \%$ and a specificity of $100 \%$ in the CSF of the same patient cohort. ${ }^{11}$ To date, Coccidioides-specific diagnostic tests have not been evaluated for use with canine CSF. The goal of the study reported here was to determine whether detection of Coccidioides antigen or antibody in canine CSF by EIA corresponded with the clinical diagnosis of CNS coccidioidomycosis in dogs and offered improved diagnosis or verification of infection.

\section{Materials and Methods}

\section{CSF samples}

Cerebrospinal fluid was collected in the course of diagnostic evaluation of dogs presenting to the neurology service at the Veterinary Specialty Center of Tucson with signs of intracranial or spinal cord disease. At this hospital, excess CSF following CSF analysis was routinely archived in cryovials and stored at $-20{ }^{\circ} \mathrm{C}$, creating a bank of specimens for study. All specimens that were collected in 2016 were tested by MiraVista Coccidioides antigen EIA (AgEIA) and MiraVista canine Coccidioides IgG EIA (AbEIA). Samples were submitted in batches of approximately 15 to 20 to MiraVista Diagnostics for the panel of tests. The frozen samples were shipped overnight in 3 batches approximately 4 months apart, with the final batch shipped in early 2017. The AgEIA was performed as previously described and was considered positive at $\geq$ $0.07 \mathrm{ng} / \mathrm{mL} .{ }^{11}$ The AbEIA for canine anti-Coccidioides IgG was performed as previously described, and results were considered positive with a result of $\geq 10$ EIA units. ${ }^{16,17}$ The investigators were blinded to patient data prior to CSF analysis.

\section{Review of records}

Records from dogs for which CSF was tested were reviewed retrospectively. The following information was collected: signalment, history, and clinical signs; results of routine CSF analysis, imaging, and serum $\mathrm{COC}$ cidioides agar gel immunodiffusion (AGID) assay; clinical diagnosis; treatment; and outcome, if available.

\section{Case definition}

Cases of CNS coccidioidomycosis (CCM) were identified by the attending board-certified neurologist at the time using a combination of history, clini- cal signs, imaging results, CSF analysis results (cell count, cell differential, and protein concentration), and serum AGID antibody results. Histologic examination was performed on available specimens, which included postmortem brain samples that had been archived in neutral-buffered $10 \%$ formalin ( $n=4$ dogs) and a biopsy specimen from $1 \mathrm{dog}$ that had undergone mass removal.

\section{Statistical analysis}

Sensitivity, specificity, and positive and negative predictive values for the AgEIA and AbEIA were performed with statistical software (Prism version 8.0; GraphPad Software Inc). ${ }^{18}$ Continuous data were assessed for normality using the D'Agostino-Pearson test, ${ }^{19}$ and the nonparametric Mann-Whitney $U$ test was performed to compare age between dogs with CCM and dogs with another neurologic disease (non-CCM). The Fisher exact test was used to compare sex distributions between the CCM and non-CCM groups. ${ }^{20}$ Results were considered significant at $P \leq 0.05$.

\section{Results}

\section{CSF samples and dogs}

Cerebrospinal fluid samples from 55 dogs and 2 cats were collected in 2016. Only samples from dogs were included in the study. Four (7\%) of the 55 samples were removed because the dogs had incomplete medical records, leaving 51 dogs in the study. Twenty-two (43\%) of these dogs were diagnosed clinically with probable or suspected CCM, while the other 29 (57\%) dogs received an alternate diagnosis (non-CCM group; Supplementary Table SI).

Dogs ranged in age from 9 months to 13 years (mean, 6.9 years; SD, 3.1 years). All but 3 dogs (2 males and 1 female) were neutered, including 27 castrated males and 21 spayed females. There were 14 mixed-breed dogs and 27 dogs of specific breeds, with more than 1 dog classified as Golden Retriever ( $n=4)$, Miniature Schnauzer (3), German Shepherd Dog (3), Greyhound (2), Russell Terrier (2), Weimaraner (2), Standard Poodle (2), and Cocker Spaniel (2). There was no difference in the median age of dogs in the CCM and non-CCM groups ( 7.25 and 7 years, respectively; $P=0.29$ ), nor did sex distribution differ between these 2 groups $(P=0.57)$.

\section{Clinical signs}

Seizures were the most common presenting complaint, seen in over half $(53 \%$ [27/51]) of the entire cohort and was the most commonly reported clinical complaint among both groups of dogs (55\% [12/22] of dogs in the CCM group; 52\% [15/29] of dogs in the non-CCM group). There was extensive overlap in clinical signs between these 2 groups. Dogs in both groups presented with lethargy, circling, ataxia, head tilt, behavior change, disturbances in vision or other cranial nerve deficits, tetra- or paraparesis, signs of neck or back pain, and head pressing. 


\section{History of coccidioidomycosis}

Six of 22 dogs (27\%) in the CCM group had historical or known concurrent coccidioidomycosis in other sites, whereas only 2 of $29(7 \%)$ dogs in the non-CCM group had any diagnosis of coccidioidomycosis.

\section{Clinical diagnosis}

Twenty (91\%) dogs in the CCM group had mass lesions or meningoencephalitis on their MRI examination, which was consistent with the literature on canine CNS coccidioidomycosis. ${ }^{3,7,8}$ Twenty-seven (93\%) dogs in the non-CCM group had MRI performed, and in $15(56 \%)$ a mass lesion or meningoencephalitis was identified. Routine CSF analysis was performed for all dogs in the CCM group and 27 (93\%) dogs in the non-CCM group ( 2 dogs were euthanized after MRI). Fifteen of 21 (71\%) dogs with CCM had pleocytosis ( 1 was excluded because of hemodilution), and 12 of $20(60 \%)$ of these dogs (2 had missing data) had an elevated CSF protein concentration (mean, $105.6 \mathrm{mg}$ / $\mathrm{dL}$; range, 11.1 to $551.2 \mathrm{mg} / \mathrm{dL}$; reference range, 14 to $30 \mathrm{mg} / \mathrm{dL})$. Pleocytosis was noted in 7 of $26(27 \%)$ dogs in the non-CCM group ( 1 was excluded because of hemodilution, and 2 were not done), while 11 of 27 (41\%) had an elevated CSF protein concentration (mean, $71.6 \mathrm{mg} / \mathrm{dL}$; range, 12 to $605.2 \mathrm{mg} / \mathrm{dL}$ ).

Serum anti-Coccidioides antibody testing by AGID assay was performed at several different commercial laboratories, with IgG titers determined for dogs with positive results. All 22 dogs with CCM had Coccidioides serology performed, of which 17 (77\%) were positive, with IgG titers ranging from 1:2 to $\geq 1: 256$. Five of the $19 \mathrm{dogs}$ in the non-CCM group that had Coccidioides serology performed had positive results, with titers ranging from 1:4 to 1:64. All but $1 \mathrm{dog}$ with CCM were treated by oral fluconazole administration at the time of diagnosis; the remaining dog died shortly after presentation. Five dogs in the non-CCM group received oral fluconazole initially because coccidioidomycosis was considered a differential diagnosis, but the medication was discontinued when an alternate diagnosis was made.

\section{CSF testing for Coccidioides antigen and antibody}

There was insufficient volume of CSF from 4 dogs to perform the AgEIA for all dogs, but all samples were tested for antibody by AbEIA. With the clinical diagno- sis used as the reference standard, sensitivity, specificity, and positive and negative predictive values were calculated for both tests (Table I). Four dogs were positive by AgEIA, and all of them had a clinical diagnosis of CNS coccidioidomycosis. None of the dogs in the non-CCM group tested positive for antigen. Overall, the AgEIA had a low sensitivity for detecting Coccidioides infection (20\%), but the specificity was $100 \%$ based on the clinical diagnosis for these cases.

A total of 14 dogs tested positive for anti-Coccidioides antibody ( $\geq 10$ EIA units) in CSF; 10 were in the CCM group, and 4 were in the non-CCM group ( 2 had brain tumors, 1 had meningitis-vasculitis, and 1 had idiopathic epilepsy). The AbEIA had low sensitivity (46\%) but high specificity (86\%). This specificity was higher than that of the serum antibody AGID assay (86\% vs $74 \%$ ).

\section{Discussion}

Results of our study confirmed that the diagnosis of CNS coccidioidomycosis in dogs presents a challenge due to nonspecific clinical signs and routine laboratory diagnostic tests as well as difficulty obtaining invasive samples for etiologic confirmation. In addition, the presence of serum antibody against Coccidioides spp in healthy dogs in a highly endemic area obfuscates interpretation of serology results, with 1 study ${ }^{21}$ demonstrating overlap in Coccidioides titers up to 1:16 in dogs with clinical and subclinical infections. ${ }^{21}$ The AgEIA and AbEIA of CSF samples showed good specificity but relatively low sensitivity in this study. The predictive value of the AbEIA suggested that in the study cohort there was a correlation between anti-coccidioidal antibodies in the CSF and a diagnosis of CCM. However, failure to find antibodies in either serum or CSF is not sufficient to exclude CNS coccidioidomycosis as a differential diagnosis, and further diagnostic investigation is essential.

A positive AgEIA result for CSF was strongly associated with a clinical diagnosis of CNS coccidioidomycosis in this cohort of dogs. This is consistent with what is reported for humans with Coccidioides meningitis. ${ }^{22}$ However, in the dogs of the present study, the test was insensitive based on the clinical diagnosis on record, which is in huge contrast to the sensitivity of the test in humans with Coccidioides meningitis (93\%). ${ }^{15,22}$ The AgEIA is also insensitive for

Table I-Utility of 3 assays for the diagnosis of CNS coccidioidomycosis in dogs with CNS disease $(n=5 I)$.

\begin{tabular}{lcccc}
$\begin{array}{l}\text { Assay } \\
\text { (sample type) }\end{array}$ & $\begin{array}{c}\text { Sensitivity } \\
(\mathbf{9 5 \%} \mathbf{~ C l )}\end{array}$ & $\begin{array}{c}\text { Specificity } \\
\mathbf{( 9 5 \% ~ C l )}\end{array}$ & $\begin{array}{c}\text { PPV } \\
\mathbf{( 9 5 \% ~ C l )}\end{array}$ & $\begin{array}{c}\text { NPV } \\
\mathbf{( 9 5 \% ~ C l )}\end{array}$ \\
\hline AgEIA (CSF) & $20(6-44)$ & $100(87-100)$ & $63(57.5-67.8)$ & $66(5 I-79)$ \\
AbEIA (CSF) & $46(24-68)$ & $86(68-96)$ & $71(48-87)$ & $68(58-92)$ \\
AGID (serum) & $77(55-92)$ & $73.7(49-91)$ & $77(61-88)$ & $74(55-86)$ \\
\hline
\end{tabular}

Data are given as percentages. A clinical diagnosis of CNS coccidioidomycosis was used as the reference standard.

$\mathrm{AbEIA}=$ Canine Coccidioides IgG enzyme immunoassay. AgEIA = Coccidioides antigen enzyme immunoassay. AGID = Agar gel immunodiffusion assay for antibody against Coccidioides spp. NPV = Negative predictive value in the studied population. PPV = Positive predictive value in the studied population. 
diagnosis of coccidioidomycosis generally in dogs using serum and urine. ${ }^{23}$ However, it is a simple and specific test if CSF is being collected as part of the workup, and this EIA merits further investigation in a prospective study, especially a study that includes confirmed etiologic diagnoses.

Compared with results for the AgEIA, results for the AbEIA corresponded more frequently with the clinical diagnosis. It is notable that the AbEIA had only moderate sensitivity for the detection of Coccidioides antibodies in CSF. Our results indicated that while AbEIA was unable to identify all dogs with CNS coccidioidomycosis, those dogs with a positive result were likely to have neurologic coccidioidomycosis.

There was good overall agreement between the presence of anti-Coccidioides antibody in CSF and serum of dogs. However, due to the retrospective nature of this study, we were unable to compare CSF and serum antibodies in this cohort. Specifically, we could not determine whether there was intrathecal production of the antibodies or transfer of serum antibodies into the CSF through a leaking blood-brain barrier. ${ }^{24,25}$ Four dogs in the non-CCM group had antiCoccidioides antibody in both serum and CSF, and it is possible that CNS inflammation in these dogs led to antibody leakage from serum to $\mathrm{CSF}^{24,25}$ One of these dogs was determined to have idiopathic epilepsy based on a clinical response to antiepileptics alone over several years, and MRI showed postictal inflammation at the time the CSF sample was collected. The other 3 dogs had histopathologic findings that confirmed neoplasia (1 dog with neuroblastoma and 1 with meningioma) or severe suppurative meningitisvasculitis and mild nonsuppurative encephalitis without evidence of an infectious agent ( $1 \mathrm{dog}$ ). The dog with meningitis-vasculitis had been diagnosed with disseminated coccidioidomycosis prior to the onset of neurologic signs and was of an age typical for idiopathic meningitis-arteritis. While several sections of the limited brain sample from this dog were histologically examined, no etiologic agent was identified. These cases underscore that the diagnosis of CNS coccidioidomycosis is more complicated in a region highly endemic for the disease.

Limitations of the present study included the retrospective design and lack of evidence available through histologic examination, cytologic examination, and microbial culture for verifying the utility of the AbEIA and AgEIA to accurately identify dogs with CNS coccidioidomycosis. Lack of proof of CNS coccidioidomycosis is typical of what practitioners face for reasons already discussed, although response to treatment of infection in the highly endemic area in which this study was performed is reasonably strong evidence. The criteria for diagnosis of Coccidioides meningitis in humans also do not include a requirement for identification of the organism in CSF or CNS tissues. ${ }^{26}$

An advantage of the study design was that the testing of CSF was performed completely without knowledge of the dogs' diagnoses. However, this ad- vantage was dampened by the lack of systematic collection of necessary data for included dogs, including the lack of serum anti-Coccidioides antibody testing, CBCs, and serum chemistry panels concurrent with the imaging and CSF analysis. Coccidioides AGID serology results were mined out of the patient record and were not necessarily performed at the same time as CSF collection and imaging. The AGID assay was also run at multiple commercial laboratories. A prospective study evaluating the use of CSF AgEIAs and AbEIAs with contemporaneous and uniform data would be useful in developing diagnostic criteria for CNS coccidioidomycosis. Performing more biopsies on suspected cases to prove the presence of Coccidioides would be ideal, but the invasiveness and cost of such procedures are likely to remain obstacles to doing so.

The spread of Coccidioides spp to the CNS is one of the most severe consequences of coccidioidomycosis in people and dogs. Although coccidioidomycosis is typically considered a disease that causes brain masses in dogs, the findings in this study suggested that meningoencephalitis without masses is common. Confirmation of a diagnosis by identification of organisms would be ideal but invasive. Coccidioidesspecific CSF tests for dogs show potential as adjunctive confirmatory diagnostics, but prospective studies to further assess these assays are needed.

\section{Acknowledgments}

Funded by the Companion Care Fund (Valley Fever Center for Excellence), University of Arizona Foundation, with in-kind support from MiraVista Diagnostics Inc.

Drs. Renschler and Wheat were both employed by MiraVista Diagnostics at the time this study was performed. The authors have no other conflicts of interest to disclose.

\section{References}

1. Nguyen C, Barker BM, Hoover S, et al. Recent advances in our understanding of the environmental, epidemiological, immunological, and clinical dimensions of coccidioidomycosis. Clin Microbiol Rev. 2013;26(3):505-525.

2. Shubitz LF. Comparative aspects of coccidioidomycosis in animals and humans. Ann N Y Acad Sci. 2007;1111:395-403.

3. Davidson AP, Shubitz LF, Alcott CJ, Sykes JE. Selected clinical features of coccidioidomycosis in dogs. Med Mycol. 2019;57(suppl 1):S67-S75.

4. Blair JE. Coccidioidal meningitis: update on epidemiology, clinical features, diagnosis, and management. Curr Infect Dis Rep. 2009;11(4):289-295.

5. Pryor WH Jr, Huizenga CG, Splitter GA, Harwell JF Jr. Coccidioides immitis encephalitis in two dogs. J Am Vet Med Assoc. 1972;161(10):1108-1112.

6. Burtch M. Granulomatous meningitis caused by Coccidioides immitis in a dog. J Am Vet Med Assoc. 1998;212(6):827829.

7. Bentley RT, Heng HG, Thompson C, et al. Magnetic resonance imaging features and outcome for solitary central nervous system Coccidioides granulomas in 11 dogs and cats. Vet Radiol Ultrasound. 2015;56(5):520-530.

8. Spoor E, Stainback L, Plummer S, Knowles K. A novel form of intracranial coccidioidomycosis is present in dogs and exhibits characteristic clinical and magnetic resonance imaging findings. Vet Radiol Ultrasound. 2019;60(1):47-55 
9. Galgiani JN, Ampel NM, Catanzaro A, Johnson RH, Stevens DA, Williams PL. Practice guideline for the treatment of coccidioidomycosis. Infectious Diseases Society of America. Clin Infect Dis. 2000;30(4):658-661.

10. Shubitz LF, Dial SM. Coccidioidomycosis: a diagnostic challenge. Clin Tech Small Anim Pract. 2005;20(4):220-226.

11. Kassis C, Zaidi S, Kuberski T, et al. Role of Coccidioides antigen testing in the cerebrospinal fluid for the diagnosis of coccidioidal meningitis. Clin Infect Dis. 2015;61(10):1521-1526.

12. Bohn AA, Wills TB, West CL, Tucker RL, Bagley RS. Cerebrospinal fluid analysis and magnetic resonance imaging in the diagnosis of neurologic disease in dogs: a retrospective study. Vet Clin Patbol. 2006;35(3):315-320.

13. Stevens DA, Martinez M, Sass G, et al. Comparative study of newer and established methods of diagnosing coccidioidal meningitis. J Fungi (Basel). 2020;6(3):125. doi:10.3390/ jof6030125

14. Jackson NR, Blair JE, Ampel NM. Central nervous system infections due to coccidioidomycosis. J Fungi (Basel). 2019;5(30):54. doi:10.3390/jof5030054

15. Bamberger DM, Pepito BS, Proia LA, et al. Cerebrospinal fluid Coccidioides antigen testing in the diagnosis and management of central nervous system coccidioidomycosis. Mycoses. 2015;58(10):598-602.

16. Holbrook ED, Greene RT, Rubin SI, et al. Novel canine anti Coccidioides immunoglobulin $\mathrm{G}$ enzyme immunoassay aids in diagnosis of coccidioidomycosis in dogs. Med Mycol. 2019;57(7):800-806.

17. Malo J, Holbrook E, Zangeneh T, et al. Comparison of three anti-Coccidioides antibody enzyme immunoassay kits for the diagnosis of coccidioidomycosis. Med Mycol. 2020;58(6):774-778.

18. Prism 9 user guide. GraphPad Software Inc; 2021. https://www graphpad.com/guides/prism/latest/user-guide/index.htm
19. D'Agostino RB, Belanger A, D'Agostino RB Jr. A suggestion for using powerful and informative tests of normality. Am Stat. 1990;44(4):316-321.

20. du Prel J-B, Röhrig B, Hommel G, Blettner M. Choosing statistical tests: part 12 of a series on evaluation of scientific publications. Dtsch Arztebl Int. 2010;107(19):343-348.

21. Shubitz LE, Butkiewicz CD, Dial SM, Lindan CP. Incidence of Coccidioides infection among dogs residing in a region in which the organism is endemic. J Am Vet Med Assoc. 2005;226(11):1846-1850.

22. Durkin M, Connolly P, Kuberski T, et al. Diagnosis of coccidioidomycosis with use of the Coccidioides antigen enzyme immunoassay. Clin Infect Dis. 2008;47(8):e69-e73. doi: $10.1086 / 592073$

23. Kirsch EJ, Greene RT, Prahl A, et al. Evaluation of Coccidioides antigen detection in dogs with coccidioidomycosis. Clin Vaccine Immunol. 2012;19(3):343-345.

24. Tipold A, Pfister H, Vandevelde M. Determination of the IgG index for the detection of intrathecal immunoglobulin synthesis in dogs using an ELISA. Res Vet Sci. 1993;54(1):40-44.

25. Tipold A, Pfister H, Zurbriggen A, Vandevelde M. Intrathecal synthesis of major immunoglobulin classes in inflammatory diseases of the canine CNS. Vet Immunol Immunopathol. 1994;42(2):149-159.

26. Galgiani JN, Catanzaro A, Cloud GA, et al. Fluconazole therapy for coccidioidal meningitis. The NIAID-Mycoses Study Group. Ann Intern Med. 1993;119(1):28-35.

\section{Supplementary Materials}

Supplementary materials are posted online at the journal website: avmajournals.avma.org. 